\title{
Low-noise microwave magnetrons by azimuthally varying axial magnetic field
}

\author{
V. B. Neculaes, R. M. Gilgenbach, ${ }^{\text {a) }}$ and Y. Y. Lau \\ Nuclear Engineering and Radiological Sciences Department, University of Michigan, Ann Arbor, \\ Michigan 48109-2104
}

(Received 28 April 2003; accepted 14 July 2003)

\begin{abstract}
A technique has been demonstrated to significantly reduce the noise in microwave oven magnetrons. The technique employs permanent magnets to generate an azimuthally varying axial magnetic field. Noise measurements are reported which show dramatic reductions in the noise of $\mathrm{kW}$ oven magnetrons operating near $2.45 \mathrm{GHz}$. The noise reduction near the carrier is some $30 \mathrm{~dB}$. Microwave sidebands are reduced or eliminated. Noise reduction occurs at all anode currents, but is particularly significant at low current near the start-oscillation condition. (C) 2003 American Institute of Physics. [DOI: 10.1063/1.1609040]
\end{abstract}

Worldwide, there exist hundreds of millions of magnetrons ${ }^{1-6}$ in microwave ovens. These magnetrons typically operate with $80 \%-90 \%$ efficiency at a frequency near $2.45 \mathrm{GHz}$. Recently, several communications systems have developed in the unlicensed, $2.4 \mathrm{GHz}$ radio spectrum: (1) Cordless telephones operating at $2.4 \mathrm{GHz},(2)$ Bluetooth, ${ }^{7}$ a wireless, short-range communication system used for computers, which operates with a spread spectrum, frequencyhopping, full-duplex signal, and (3) IEEE $802.11 \mathrm{~b}$ and $802.11 \mathrm{~g},{ }^{8}$ a complementary code keying-orthogonal frequency division multiplexing system used for computer wireless local area networks, operating in the frequency range from $2.4 \mathrm{GHz}$ to $2.4835 \mathrm{GHz}$. Since these communication systems occupy the same region of the microwave spectrum utilized by microwave ovens, there exists significant potential for interference from noisy magnetrons. ${ }^{9}$

The noise generation mechanisms of linear electronbeam devices are well known. ${ }^{3}$ On the other hand, noise generation mechanisms in crossed-field devices are not presently understood and predictive computational calculations do not exist. Methods of noise suppression in crossed-field devices have not previously been practically realized. Previous researchers ${ }^{5,6}$ have shown that cathode heater conditions played a role in the noise of microwave magnetrons. Noise in crossed-field amplifiers (CFAs) ${ }^{10-13}$ also significantly limits the signal-to-noise ratio in military radars. In this article, we present spectral measurements which definitively demonstrate that microwave magnetron noise is significantly reduced by the imposition of an azimuthally varying axial magnetic field. Further, this technique has been shown to be effective in noise reduction regardless of the magnetron current or age.

The experimental configuration is depicted in Fig. 1. Standard microwave oven magnetrons have been employed, from different manufacturers (Panasonic, Toshiba). These magnetrons operate at a center frequency of about $2.45 \mathrm{GHz}$ at power levels of about $700-800 \mathrm{~W}$. A dc power supply is utilized at typical cathode voltage of about $-4 \mathrm{kV}$ and anode currents up to $300 \mathrm{~mA}$. The microwave power is dissipated

a)Electronic mail: rongilg@umich.edu in a water load and a fraction of the power is split by a directional coupler into an Agilent spectrum analyzer. Cathode heater power is kept nearly constant for the experiments presented here.

Figure 2(a) depicts the standard magnet configuration in a typical oven magnetron. Two cylindrical magnets are utilized at opposite ends of the magnetron with a magnetic yoke providing the flux return path. The standard magnets have a magnetic field on the face of about $1 \mathrm{kGauss}$ with an axial magnetic field at the center of the magnetron of about 1.7 kGauss. The modification of the technique of the magnetic field for low noise operation is depicted in Figs. 2(b) and 2(c). Four permanent magnets (about $3.5 \mathrm{kGauss}$ on the face) have been added to the outside of one of the standard magnets to locally perturb the magnetic field in such a way as to cause the total axial magnetic field outside of the magnetron cavities to vary azimuthally by about $50 \%$. As seen in Fig. 2(c), the perturbing magnets neither need to be identical in physical size, nor do they need to be symmetric or periodic in the azimuthal direction. Fewer than four perturbing magnets show less reduction in power, but less-effective noise suppression; the correlation between these parameters is an area of future study.

Figure 3(a) shows an experimental spectrum for an aged magnetron with the standard magnetic field. The data exhibit a broad noise spectrum, which begins some $-30 \mathrm{~dB}$ below the carrier. There are typically two components to this noise: The component close to the carrier and the sidebands centered $120 \mathrm{MHz}$ from the center frequency. Note that the more widely spaced sidebands (called "spurs" in literature) in the

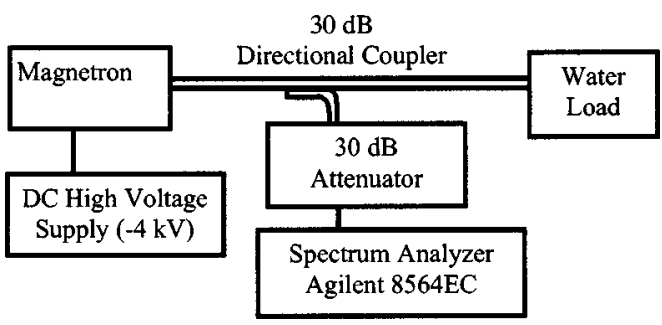

FIG. 1. Experimental configuration for oven magnetron spectrum analysis. 

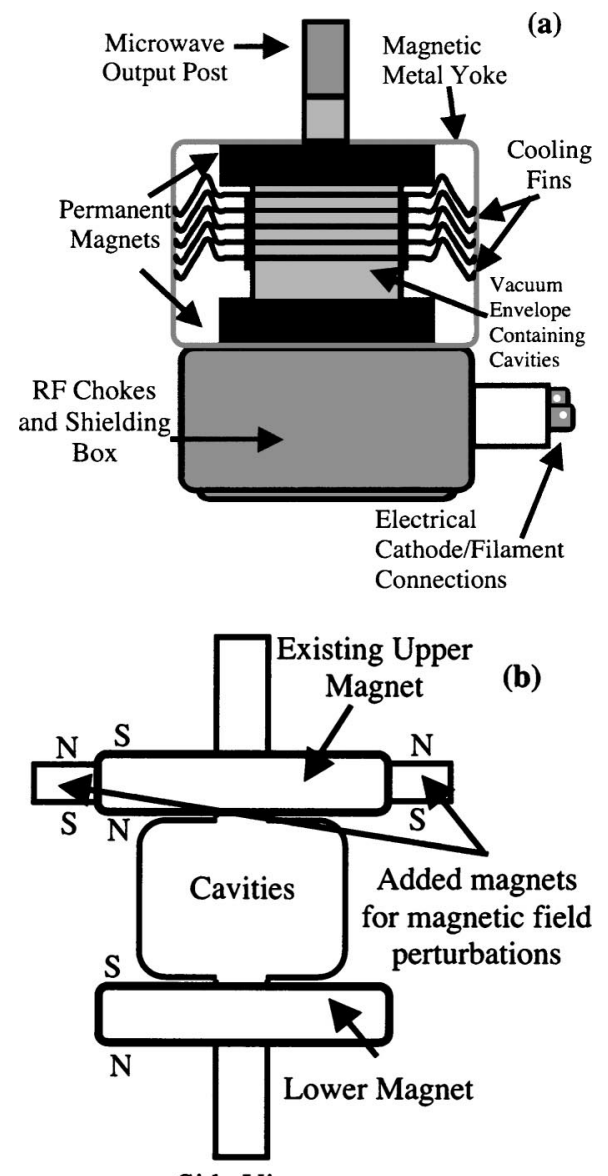

Side View

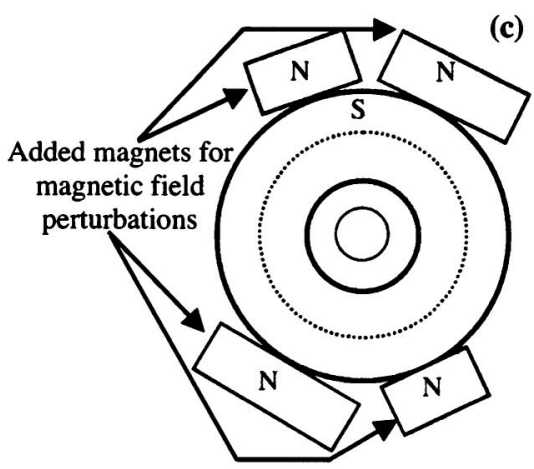

Top View

FIG. 2. (a) Typical oven magnetron configuration showing upper and lower annular, permanent magnets: (b) Side and (c) top views of magnetic field configuration for typical azimuthally varying axial magnetic field utilized for noise reduction experiments.

noise spectrum are believed due to the recirculating electrons. ${ }^{14}$

Figure 3(b) depicts the microwave spectrum for the same aged magnetron in which four permanent magnets have been added, as in Figs. 2(b) and 2(c). The spectrum for the azimuthally varying axial magnetic field is extremely narrow and completely free of the noise present in Fig. 3(a). Older magnetrons exhibit a noisier spectrum than fresh magnetrons, probably due to cathode conditioning and the increased gas pressure due to vacuum leaks over time. However, it should be noted that the same noise reduction effect has been measured for fresh magnetrons when the magnetic field is modified, as shown in Fig. 4. This technique reduces
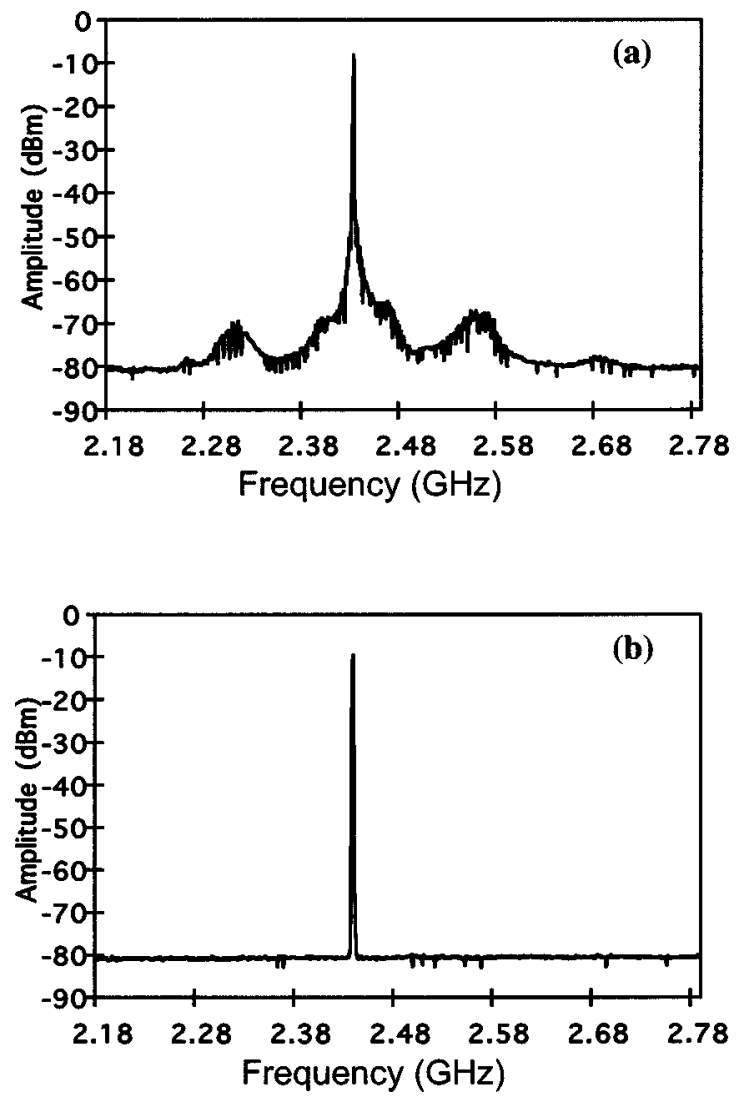

FIG. 3. Microwave spectra for aged Panasonic 2M167A-M10 magnetron at anode current of $226 \mathrm{~mA}$ : (a) With standard magnetic field and (b) with azimuthally varying axial magnetic field.
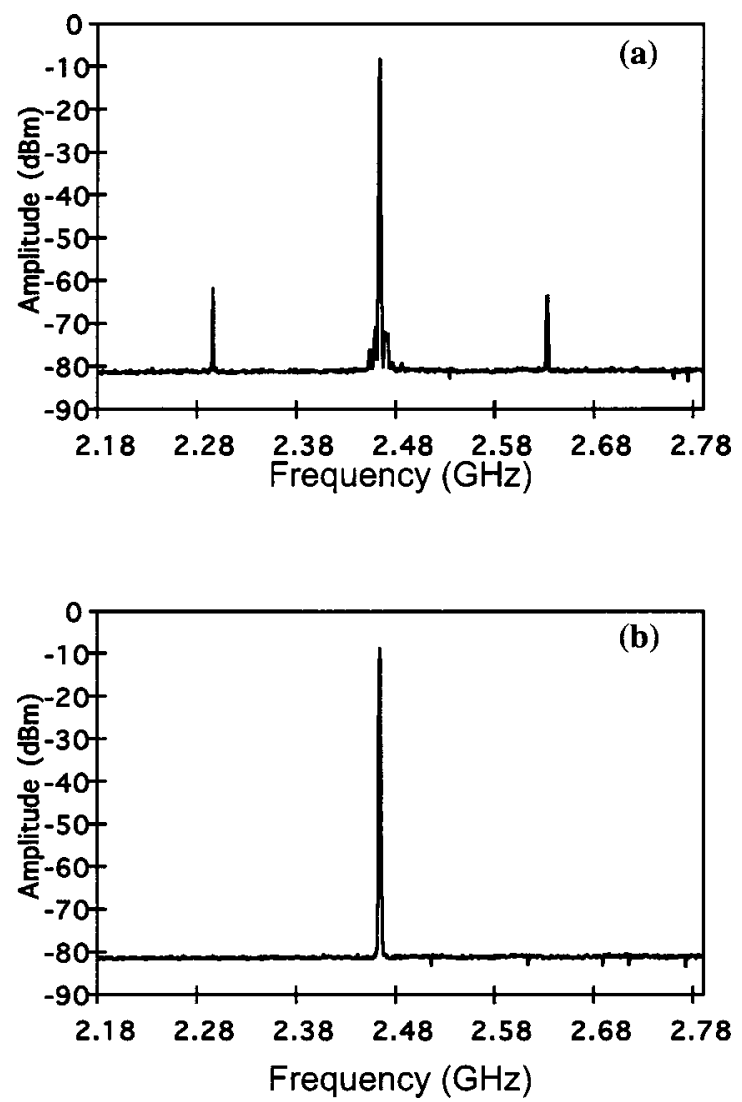

FIG. 4. Microwave spectra for Toshiba fresh $2 \mathrm{M} 172 \mathrm{~J}(\mathrm{E})$ magnetron at anode current of $215 \mathrm{~mA}$ : (a) With standard magnetic field and (b) with azimuthally varying axial magnetic field. 

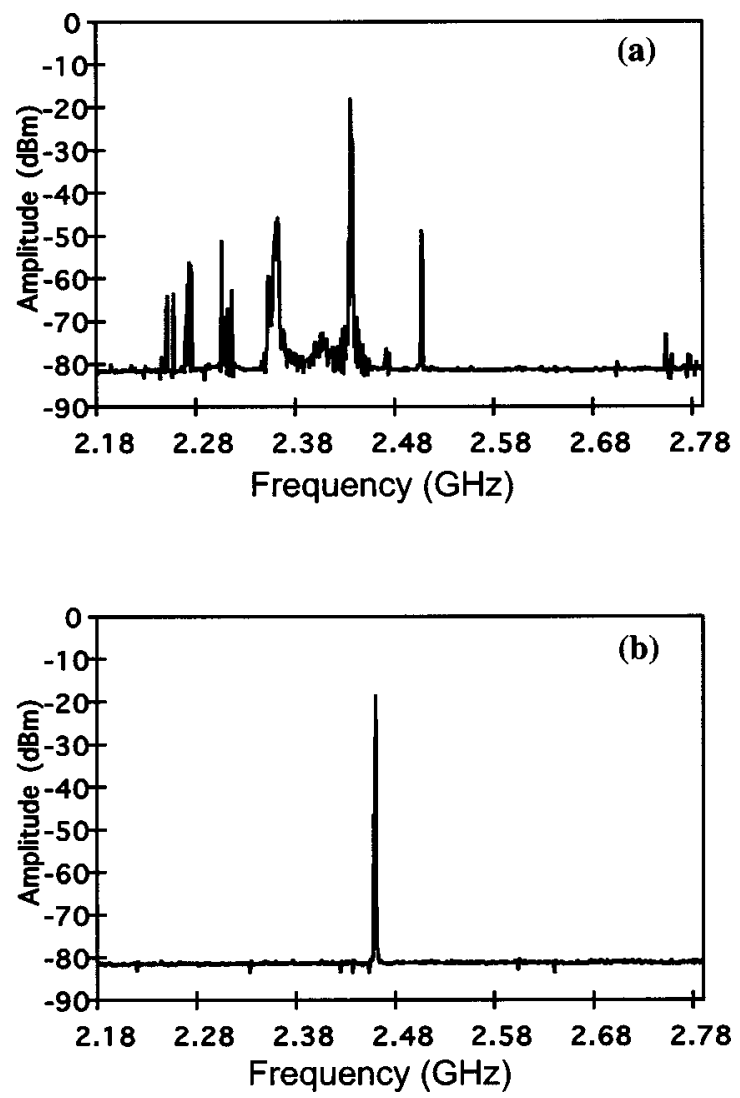

FIG. 5. Microwave spectra near start-oscillation condition for fresh Toshiba 2M172 J(E) magnetron at anode current of $33 \mathrm{~mA}$ : (a) With standard magnetic field and (b) with azimuthally varying axial magnetic field.

the microwave power for fresh magnetrons by about $10 \%$ and for old magnetrons by about $20 \%$. Since the currentvoltage characteristics are changed (lower impedance) by the added magnets, the efficiency of fresh magnetrons is reduced by $3 \%-7 \%$ and old magnetrons show an efficiency reduction of about $10 \%$.

This type of noise-free microwave spectrum has been observed at all magnetron currents above start oscillation, but is particularly effective at low magnetron currents, near the start-oscillation condition. Figure 5(a) represents the extremely noisy microwave spectrum at a low magnetron current of $33 \mathrm{~mA}$, close to the start-oscillation condition, with the standard magnetron magnets. When the azimuthally varying axial magnetic field is added, the spectrum is modified to the single spectral line depicted in Fig. 5(b). This improvement in magnetron startup noise would be particularly important in microwave ovens, which operate with a voltage-doubled half-wave rectified sinewave voltage source and, hence, are repetitively sweeping through the startup phase at $60 \mathrm{~Hz}$.

The physical mechanism of the noise reduction is not completely understood; one candidate is the perturbing effect on recirculating electrons in the magnetron. Calculations show that the recirculation time for electrons in the oven magnetron is consistent with the $120 \mathrm{MHz}$ spacing of the sideband spurs [Fig. 3(a)]. Further research is directed toward identifying these noise reduction mechanisms.

In summary, a simple magnetic field modification is shown to significantly reduce the microwave noise in magnetrons by an azimuthally varying axial magnetic field. This noise reduction technique is effective in fresh or aged magnetrons and cleans the spectrum especially well during the start-oscillation condition. Extension of this concept to CFAs could also have an enormous impact on the signal-to-noise ratio in radars.

Note added in proof: Additional experiments and simulations show that the most rapid startup occurs with $n$ magnetic perturbation periods, so as to prebunch the electrons, where $n$ is the number of electron spokes in the operating magnetron mode. Experiments again show low-noise operation with this rapid startup.

This research was supported by the DUST (S\&T) under the Innovative Microwave Vacuum Electronics MURI Program managed by the Air Force Office of Scientific Research under Grant No. F49620-99-1-0297. Sponsorship was also received from the Air Force Office of Scientific Research under Grant No. F49620-00-1-0088. The authors appreciate assistance from Michael Jones.

${ }^{1}$ G. B. Collins, Microwave Magnetrons (McGraw Hill, New York, 1948); J. C. Slater, Microwave Electronics (Van Nostrand, New York, 1950).

${ }^{2}$ J. Benford and J. Swegle, High Power Microwaves (Artech House, Boston, 1992)

${ }^{3}$ A. S. Gilmour, Jr., Microwave Tubes (Artech House, Norwood, MA, 1986).

${ }^{4}$ J. M. Osepchuk, IEEE Trans. Microwave Theory Tech. 50, 975 (2002).

${ }^{5}$ W. C. Brown, Proceedings of the First International Workshop on Crossed-Field Devices, edited by D. Chernin and Y. Y. Lau, Ann Arbor, Michigan, 15-16 August 1995, p. 178, available at http://wwwners.engin.umich.edu/labs/plasma/.

${ }^{6}$ J. M. Osepchuk, Proceedings of the First International Workshop on Crossed-Field Devices, edited by D. Chernin and Y. Y. Lau, Ann Arbor, Michigan, 15-16 August 1995, p. 159, available at http://wwwners.engin.umich.edu/labs/plasma/.

${ }^{7}$ See, e.g., http://www.bluetooth.com/

${ }^{8}$ See, e.g., http://grouper.ieee.org/groups/802/11/

${ }^{9}$ For an audio demonstration of cordless phone interference from microwave oven radiation, see http://www-ners.engin.umich.edu/labs/plasma/

${ }^{10}$ H. L. McDowell, IEEE Trans. Plasma Sci. 30, 980 (2002).

${ }^{11}$ R. MacGregor, C. Chan, J. Ye, and T. Ruden, IEEE Trans. Electron Devices 41, 1456 (1994).

${ }^{12}$ D. P. Chernin, IEEE Trans. Electron Devices 43, 2004 (1996).

${ }^{13}$ H. L. McDowell, Proceedings of the First International Workshop on Crossed-Field Devices, edited by D. Chernin and Y. Y. Lau, Ann Arbor, Michigan, 15-16 August 1995, p. 217, available at http://wwwners.engin.umich.edu/labs/plasma/.

${ }^{14}$ K. Yamamoto, H. Kuronuma, T. Koinuma, and N. Tashiro, J. Microwave Power 16, 271 (1981). 